\title{
Quelques réflexions à la lecture de l'Éloge de Foscolo (1827) de Dionysios Solomos et de sa Correspondance (1816-1856)
}

Quelles élites pour quel peuple dans les îles Ioniennes au début du $\mathrm{XIX}^{\mathrm{e}}$ siècle?

What Elites for what People in the Ionian Islands in the Early 19th Century?

Some Reflections on Foscolo's Eulogy (1827) and Dionysos

Solomos'Correspondance (1816-1856)

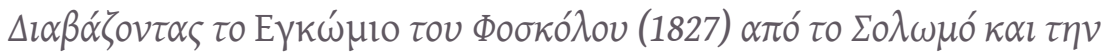

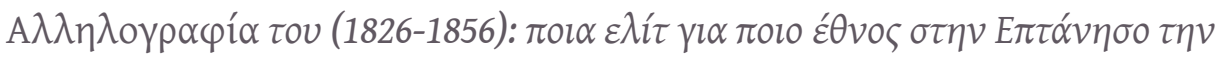

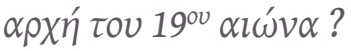

\section{Anastasia Danaé Lazaridis}

\section{OpenEdition}

\section{Journals}

Édition électronique

URL : https://journals.openedition.org/ceb/5642

DOI : $10.4000 /$ ceb.5642

ISSN : 2261-4184

\section{Éditeur}

INALCO

Édition imprimée

Date de publication : 1 mars 2015

ISBN : 978-2-85831-224-5

ISSN : 0290-7402

Référence électronique

Anastasia Danaé Lazaridis, «Quelques réflexions à la lecture de l'Éloge de Foscolo (1827) de Dionysios Solomos et de sa Correspondance (1816-1856) », Cahiers balkaniques [En ligne], Hors-série | 2015, mis en ligne le 13 janvier 2016, consulté le 06 juillet 2021. URL : http://journals.openedition.org/ ceb/5642 ; DOI : https://doi.org/10.4000/ceb.5642

Ce document a été généré automatiquement le 6 juillet 2021.

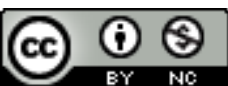

Cahiers balkaniques est mis à disposition selon les termes de la Licence Creative Commons Attribution - Pas d'Utilisation Commerciale 4.0 International. 


\section{Quelques réflexions à la lecture de l'Éloge de Foscolo (1827) de Dionysios Solomos et de sa Correspondance (1816-1856)}

Quelles élites pour quel peuple dans les îles Ioniennes au début du XIX ${ }^{\mathrm{e}}$ siècle?

What Elites for what People in the Ionian Islands in the Early 19th Century?

Some Reflections on Foscolo's Eulogy (1827) and Dionysos

Solomos'Correspondance (1816-1856)

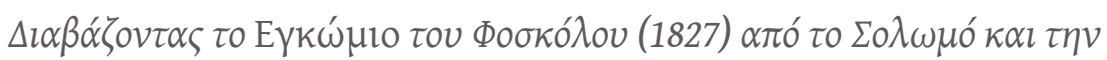

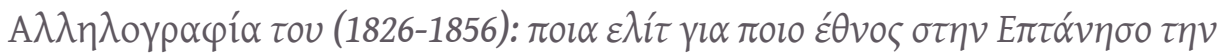

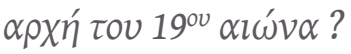

Anastasia Danaé Lazaridis

\section{L'Oraison funèbre}

1 En octobre 1827, Dionysios Solomos, d'après sa Correspondance (PoLITIS, 1991, 134-136), faisait porter ce billet à son ami Spyridon Pilicas, à Zante ${ }^{1}$ :

Cher toi, fais ton possible pour venir à midi, ou à une heure, pour écrire quelque chose, car ainsi nous ferons plus vite. C'est mieux que je te dicte en vitesse, comme nous avons fait hier, et qu'ensuite tu mettes au propre chez toi. Ce sera bien que tu n'ailles pas déjeuner à la maison, mais que tu déjeunes ici, pour ne pas perdre de temps. Eh! N'y manques pas; de toute façon, envoie-moi une réponse par le porteur $<$ de ce billet $>$.

2 Vu la date du texte et la sensation de travail dans l'urgence, il s'agit très probablement de la rédaction de l'oraison funèbre de Ugo Foscolo, que Solomos prononça le 19 novembre 1827 (PoLITIS, 1991, 138) dans la cathédrale catholique de la ville de Zante, 
en hommage à son illustre compatriote décédé le 10 septembre, en exil à Londres². Le manuscrit de 44 pages qui en contient la copie (ms. de Zante, Loge maçonnique $\mathrm{n}^{\circ} 4$ ), sans doute de la main de Spyridon Pilicas, est aujourd'hui conservé au Musée Solomos

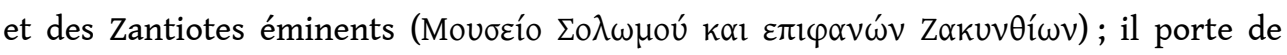
nombreuses corrections et biffures de la main de Solomos, ainsi que l'indication «Orazione in morte di U. Foscolo recitata nella Catt. di Zante nella state 1827 ». Le texte original en italien et une belle traduction grecque ont été publiés par Linos Politis, respectivement dans le deuxième volume et son Annexe des Euvres complètes de Solomos (Politis, $1955^{1}$ et $1960^{1}$ ), mais l'Éloge fut traduit plusieurs fois en grec dès 1901, puis en français par Joseph Peretti en 1957 (PoLITIS, 1960 et 19913, 132) - traduction qu'il serait bon de reprendre aujourd'hui. Politis mentionne encore l'édition de Carlo Brighenti (FERRARE, 1929), dont l'introduction et les notes ont été précieuses à l'éditeur grec (et à son collaborateur, G. N. Politis) pour traduire ce «texte italien extrêmement difficile» (PoLITIS, 1991³ 1 132). Quant à Spyridon Pilicas (1805-1861), né à Constantinople et installé tôt à Zante, promis à une longue carrière de juriste, professeur, aréopagite et même ministre du jeune État grec, il fait alors partie de ce groupe d'amis qui ont été les « secrétaires » de Solomos à son retour d'Italie et durant la période zantiote de sa production poétique.

Deux autres missives de novembre 1827 (PoLITIS, 1991, 136-141) adressées à Giorgio de Rossi se rapportent à la rédaction de l'oraison funèbre de Foscolo et témoignent du même souci : gagner du temps, mais exceller dans cet exercice de rhétorique destiné à une célébration publique, ce qui ne constitue pas l'aspect le plus négligeable de l'entreprise.

4 Giorgio de Rossi (1780/81-1860 ?), issu d'une famille catholique de Zante, est à l'époque - surtout entre 1824 et 1828 - avec Ludovico Strani (1794-186 ?), un ami très proche de Solomos et son principal «secrétaire-copiste». À Bologne de 1808 à 1810, il s'était lié d'amitié avec Ugo Foscolo et semble avoir assisté à sa célèbre leçon inaugurale Dell'origine e dell'ufficio della letteratura, prononcée en janvier 1809 suite à sa nomination à la chaire de rhétorique de l'Université de Pavie. À Zante, où il occupa diverses hautes fonctions dans l'administration publique, de Rossi fut dénoncé aux autorités anglaises et arrêté par la police en 1821, car il faisait circuler le rapport signé par lui et d'autres notables de l'île - même par Solomos - adressé au roi Georges IV pour demander des réformes libérales et notamment la révision de la très autoritaire "Constitution" de 1817 de Lord Maitland. Évadé, de Rossi trouva refuge en Angleterre et ne put rentrer dans son île natale qu'en 1824. Quelques mois plus tard, il épousa la sœur de Ludovico Strani, Susanna, et le couple entretint des relations très affectueuses avec le poète. Par la suite, pour des raisons encore assez mystérieuses, les rapports entre les deux amis se sont considérablement refroidis.

Solomos semble avoir souhaité dédier à Giorgio de Rossi l'Ode à Byron, restée inachevée, dont la fin de la première élaboration date d'octobre 1827 - presque en même temps que la rédaction de l'Éloge à Foscolo - et à Ludovico Strani le Dialogue (1824); c'est en tous cas chez les Strani que fut écrit en 1823 l'Hymne à la Liberté, et c'est grâce à cet ami que furent publiées en 1822 à Corfou les Rime improvvisate, avec une chaleureuse dédicace à Ugo Foscolo. Strani fut à Zante consul de Venise, où il s'installa en 1825 et où il mourut. Fervent défenseur de l'Insurrection grecque, il fut emprisonné en octobre 1821 suite aux événements d'Ypsolithos ${ }^{3}$. Apparemment, Solomos n'eut plus de 
contacts avec lui après son départ de l'île, peut-être à cause d'un litige pour des questions d'argent (POLITIS, 1991, 101-111 ; KAPSASKIS, 1998, 149-155, 156, 185, 344-345).

6 Les deux billets de Solomos à de Rossi témoignent de l'angoisse de perfection du poète, qui jouit alors d'une certaine notoriété dans son île natale, mais également en Europe, pour son Hymne à la Liberté déjà publié en anglais et en français (Literary Gazette, Londres 1824 ; Chants populaires de la Grèce moderne de Claude FAURIEL, 1824) et en italien à Missolonghi (1825). Jacovaky Rizo Néroulos, malgré quelques réserves, en fit une présentation élogieuse dans son Cours de littérature grecque moderne $\left(1828^{2}, 163\right)$.

7 Les hautes exigences et l'insatisfaction du poète à l'égard de lui-même et de son texte sont patentes dans le premier billet :

Hier soir, faisant à voix très basse ces essais que tu me disais, je vis, l'esprit tranquille, qu'à l'exception de trois ou quatre passages de l'oraison (qui en vérité sont de grande et quasi unique importance) tout le reste de la rédaction, pour des raisons étrangères à la brièveté de cette petite lettre, est (je te prie de ne pas t'effrayer), est à refaire. Mais avant de me donner cette peine, ce serait bien de savoir si tu peux, grâce à ton ingéniosité, trouver des arguments [...] pour prolonger le délai de quelques jours, et cela sans que tu ne me compromettes en aucune manière. [...] parce que je me sens plus apte à réciter mal une chose bonne qu'à réciter bien, en imposteur, une chose mauvaise. Voilà que je me remets à te dire que celle-ci est mauvaise, mais le temps m'est bref pour te faire comprendre en quel sens ; et peu de gens s'en apercevront [...].

8 Dans le second billet, ses aveux sur la qualité et les conditions de son travail dénotent sa perception du labeur intellectuel et la conscience de soi développée par le poète :

[...], car moi je ne t'ai pas écrit que l'oraison est mauvaise, mais que ces quelques parties je les voudrais modifiées. Et ne crois pas que ce soit bien pour moi que tu m'ôtes tout espoir de temps au-delà de sam<edi>, et tes arguments ne tiennent pas, et voici pourquoi. En premier lieu, les trois jours et les trois nuits dont tu parles seraient valables au cas où il s'agirait de quelque cheval malingre qui travaillerait

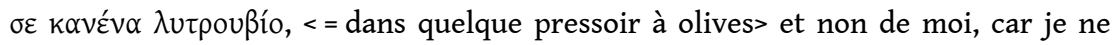
peux écrire que pendant très peu d'heures par jour. En second lieu (et cela est plus important, je te prie d'y réfléchir), quand je vois devant moi une heure de plus, c'est avec une disposition d'âme différente que je profite de l'heure présente, laquelle, sinon, deviendrait angoissée et perturbée.

9 Il est indéniable que l'effort fourni par Solomos pour la rédaction de l'Éloge fut considérable, et que le temps était compté. Iacovos Polylas, le premier éditeur en 1859 des Evplбкó $\mu \varepsilon v \alpha$ (Euvres subsistantes), s'en fait l'écho dans ses Prolégomènes (v. PoLITIS, $1948^{1}$ et $\left.1979^{4}, 24\right)$ :

L'année suivante (1827) mourut en Angleterre son glorieux compatriote, Foscolo. Dès que la nouvelle parvint à Zante, Solomos composa tout de suite et prononça dans l'église des Catholiques ce discours funèbre où sa puissance rhétorique ne paraît pas inférieure à celle de sa poésie. Il fit une telle impression sur l'auditoire, que le lieu sacré n'a pas empêché les gens d'exprimer leur enthousiasme par de vifs applaudissements. Et si la beauté de la Parole enchante aussi le lecteur impartial, ses auditeurs éprouvaient encore un autre sentiment très rare; car, alors que Solomos, faisant l'éloge de l'homme éminent, se présentait lui aussi en enthousiaste fervent des lettres, en extrême zélote des devoirs élevés du philologue et en ennemi mortel de l'hypocrisie, de la médiocrité et de la fausse sagesse - ses compatriotes savaient que ces paroles-là n'étaient pas des exagérations rhétoriques, mais le pur épanchement de la bonté et de l'incandescence de son âme.

10 Polylas ne manque pas de souligner l'importance de l'Éloge pour l'image publique de son auteur et l'affirmation de sa propre excellence; le disciple fidèle avait bien senti 
que le juste hommage à Foscolo constituait en même temps pour Solomos une belle occasion de donner la mesure de la reconnaissance dont il pouvait jouir auprès de ses compatriotes, une dizaine d'années à peine après son retour à son île. Plaçant le maître vénéré sous la triple lumière de l'homme de lettres accompli, du poète (" philologue ») conscient de sa haute mission et ennemi naturel du faux dans l'art et la science, de l'homme distingué pour son âme ardente, pour sa «bonté " par-delà l'art et le savoir le mot utilisé ici, $\alpha \gamma \alpha \theta o ́ s$, renvoie à l'idéal antique du citoyen - Polylas traçait le

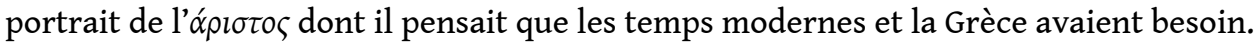

11 Pièce de circonstance d'un genre rhétorique bien déterminé, de facture très classique, l'Oraison funèbre de Foscolo fut sans doute pour Solomos un exercice d'excellence obligé, exécuté dans les règles de l'art et brillamment réussi.

Mais au-delà des questions de genre et d'école, ce texte, qui appartient de droit aux lettres italiennes, mérite de retenir l'attention dans le cadre d'une réflexion plus large sur les élites et les attitudes mentales qui permettent de mieux cerner le paysage de l'hellénisme moderne en ce premier tiers $d u \mathrm{xIX}^{\mathrm{e}}$ siècle, une période traversée de bouleversements sociopolitiques et économiques majeurs, qui vit la marche vers l'indépendance grecque s'accélérer de façon impressionnante. Le séisme de la Révolution Française, les guerres napoléoniennes et la nouvelle carte de l'Europe issue du congrès de Vienne en 1815 et de l'institution de la Sainte-Alliance, l'instabilité politique des îles Ioniennes qui, après avoir caressé un doux, mais bref rêve d'indépendance, ont connu la soumission au "protectorat» anglais dès 1815, les prémisses du Risorgimento et les désillusions du patriotisme italien (Foscolo en fit largement les frais), la guerre d'Indépendance grecque sur le point d'aboutir avec l'inattendue, mais décisive bataille de Navarin (en octobre 1827 !)... Cette course folle des événements, des personnes comme Foscolo ou Solomos - de vingt ans son cadet l'ont vécue et en ont subi plus ou moins fortement les conséquences, tant au niveau collectif qu'individuel. Dans l'Éloge de Foscolo, derrière la célébration conventionnelle de l'homme de lettres, transparaît la trame sombre de l'Histoire où la destinée des peuples se confond avec celle des individus, tant les fils de l'une et de l'autre s'entretissent.

13 Un tel témoignage réjouira l'historien des idéologies, ainsi que l'a souligné Georges Duby dans son Histoire sociale et idéologie des sociétés $(1974,212)^{4}$. Tout en faisant l'éloge de l'homme éminent qui avait vu le jour à Zante, mi-Grec mi-Italien et si distingué dans les lettres italiennes et la culture européenne, Solomos avait l'occasion de réfléchir, à un moment crucial aussi bien pour son propre itinéraire de noble heptanésien de culture italienne et de conscience de plus en plus grecque, que pour la Grèce et l'ensemble de l'Europe, à un modèle d'excellence qui avait fait ses preuves et traversé les frontières, alliant le citoyen et l'homme d'action à l'intellectuel, l'érudit et le poète. La mort de Foscolo en apatride solitaire ajoutait une dimension symbolique au personnage déjà emblématique qu'il fut de son vivant.

14 Le soin que Solomos mit à rédiger et à parfaire ce texte, la complexité de sa facture, laissent en outre penser qu'il souhaitait le publier - à Corfou, en Italie ou ailleurs en Europe - pour se mesurer à armes égales depuis sa petite île de Zante avec les intellectuels du monde "civilisé ». Cette ambition était sans doute partagée par son réseau d'amis intimes qui désiraient promouvoir une nouvelle figure de l'intelligentsia locale et de leur cercle, capables de rivaliser avec les meilleurs esprits européens au 
moins dans le domaine d'une culture à la fois cosmopolite et nationale jouissant d'un important prestige symbolique.

\section{Un portrait de l'excellence - Un portrait en miroir ?}

15 L'apostrophe aux auditeurs qui ouvre le discours, les désignant comme des pèlerins sur le chemin de la vie - image toute foscolienne - fait appel simultanément à leur esprit et à leur cœur ; la disparition des génies sublimes (sublimi intelletti) fait naître en ceux qui ressentent une «douleur généreuse " le désir d'exprimer leur chagrin de voir ainsi diminué le nombre déjà infime des hommes destinés à modérer les motifs infinis de la misère humaine ; c'est le cas des habitants de Zante, qualifiés de «bons » (quei buoni), qui ont appris par la lecture des journaux apportant les nouvelles du monde sur leur île certes petite, néanmoins réputée comme "gentille» auprès des étrangers (questa isoletta, che ha nome di gentile presso gli estrani), la mort de Ugo Foscolo.

Les "génies sublimes", mais mortels, ayant «reçu une plus grande part de cette lumière divine qui pénètre merveilleusement toute la Création ", leur excellence semble être une sorte de mission en ce monde: atténuer l'infélicité humaine. Pour Solomos, Foscolo compte parmi ces génies, selon cet ordre divin plus qu'humain.

17 À la tristesse des savants européens, au deuil des Académies italiennes, le "pauvre parterre de fleurs " (questa povera aiuola) ${ }^{5}$ de la terre natale de Foscolo fait écho selon ses moyens, par un deuil public et spontané, empreint de la fierté d'avoir donné naissance au poète. Zante lui était redevable de l'amour qu'il ne cessa de lui porter en toute occasion, notamment dans ses vers immortels et sa sublime composition des Grazie. En effet, dans ce poème inachevé où, sous le voile du mythe antique, il entendait laisser transparaître sa vision de l'ennoblissement du genre humain, il adressait d'abord un salut à la "Patrie», avant de se sentir digne de converser avec les vénérables divinités célébrées sous sa plume.

18 Par une allusion quasi détournée aux Ultime lettere di Jacopo Ortis (1802), œuvre de fiction et d'idéologie imprégnée par une sorte d'apologie du suicide, Solomos semble

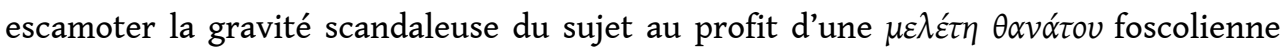
toute tournée vers la vie. Naturellement doué de curiosité insatiable à l'égard de tout ce qui l'entourait et de toutes les personnes qu'il rencontrait, Foscolo était abondamment doté des deux caractéristiques de l'excellence selon Solomos : le grand génie et l'étude constante. Ses maîtres en Italie ne tardèrent pas à reconnaître, associés à un esprit très «capable» de nature (intelletto molto capace), ses dons particuliers et son mérite personnel ; Melchiore Cesarotti, Vincenzo Monti et Giuseppe Parini virent en lui un génie promis à une grande destinée et l'encouragèrent à gravir les sommets. Ils s'émurent en songeant à sa terre natale, alors couverte de cendres, mais d'où la flamme pouvait à nouveau jaillir et prouver qu'elle était encore la terre qui transmit au monde « l'image éternelle du Beau » : la Grèce, par l'intermédiaire de Zante.

Couvert de gloire grâce à ses talents littéraires, Foscolo fut aussi un véritable érudit: l'édition de la Chioma di Berenice (1803), sa leçon inaugurale à l'Université de Pavie sur l'origine et la mission de la littérature (1809), la méthode critique qu'il suivit dans ses leçons par la suite - elle lui permettait en particulier, en examinant le statut des lettres, des sciences et des arts, des coutumes, de la religion, des institutions politiques et de la philosophie de chaque époque, de « démontrer si l'auteur était au-dessous, au même 
niveau, ou au-dessus de son siècle » (Orazione, 1979, 192) - de même que ses essais sur Dante, Pétrarque, Boccace, ou sa dissertation sur le digamma éolien et ses écrits datant de l'exil en Angleterre (dès 1816), donnent la preuve de l'étendue de sa connaissance des auteurs grecs et latins, de sa capacité de jugement et de son époustouflant esprit critique, grâce auquel il se fit un plaisir de railler non seulement les faux-semblants, mais aussi les savants pour qui l'érudition comptait comme but en soi, et non comme moyen d'atteindre la sagesse.

20 À Foscolo poète, Solomos rend un hommage très intéressant, en consacrant une longue analyse littéraire, fine et originale, à sa composition majeure qui lui valut la « somma gloria »: le poème des Sepolcri (1806). Il fit surtout remarquer que les sépultures y ont été envisagées d'un point de vue politique, dans le but de raviver l'émulation parmi les Italiens par des exemples de peuples qui honorèrent la mémoire et les tombeaux des grands hommes, et de les inciter à faire de leur vie œuvre utile pour la patrie. Sans visées vers la vérité religieuse, morale, politique et physique, et sans talent, les hommes méritent le bannissement de la cité, où ils ne sont que des oisifs ennuyeux et ridicules. Dire la vérité, défendre la justice en toutes circonstances - quitte à vilipender son propre pays, comme Foscolo l'a fait à l'instar des tout grands, Dante, Pétrarque, Boccace et l'Arioste - tel serait l'apport du poète à la civilisation d'une Italie déjà civilisée.

Contraint à l'exil, Foscolo chercha refuge en Angleterre : choix doublement judicieux, selon Solomos, car dans son pays la gloire des arts se trouvait mêlée à la honte d'un certain esclavage, et parce que les Anglais, jouissant de bonnes institutions politiques, se distinguaient avec succès dans les arts, le savoir et les armes. Ici, l'orateur s'empressait de préciser qu'il entendait par «bonnes » les institutions avec lesquelles ils se gouvernaient seulement eux-mêmes, faisant ainsi une brève, mais très nette allusion au gouvernement britannique des îles Ioniennes qu'il a connu - sinon subi lui-même, dès son retour à Zante en 1818.

Comparant enfin Foscolo à l'autre enfant terrible du siècle qu'était le noble, très libéral et très philhellène lord Byron, Solomos les déclare à la fois semblables et dissemblables : de statut social différent, ils avaient en commun une célébrité rapide, un esprit ardent, la force du courage, l'amour magnanime de la Liberté comme source des vertus civiques, l'extraordinaire, spontanée, fulgurante et indomptable éloquence, la finesse de la raillerie contre les pédants, une façon bien à eux de proclamer les vérités utiles dans les lettres ou en politique, ce qui leur valut de solides inimitiés dans leurs pays respectifs.

\section{La fiction à l'œuvre}

À deux reprises dans l'Oraison, Solomos, mû par son propre génie littéraire, laisse libre cours à son imagination. Évoquant d'abord la leçon inaugurale de Foscolo à l'Université de Pavie, où lui-même suivit des cours de droit entre 1816 et 1818, il dresse, presque en témoin oculaire, aussi bien le décor du lieu que le portrait psychologique de l'orateur : dans une salle immense où se trouvaient nombre de savants qui auraient pu être ses pères, des hommes de toute condition et de tout âge et quelques deux mille élèves, Foscolo a été écouté dans un silence absolu ; deux heures plus tard, ce fut une explosion d'applaudissements et de cris d'émotion intense qui se déversèrent à l'extérieur, gagnant toutes les rues de la ville. Un « jeune homme » (il giovinetto) qui l'avait écouté 
se mit à parcourir alors ces mêmes rues, se demandant si lui-même serait apte à la littérature utile, se disant qu'il fallait beaucoup d'étude pour répondre aux besoins de sa patrie ; qu'il devait aimer le Vrai, surtout ne pas faire de la parole un instrument de flatterie, respecter la morale et la religion, devenir toujours meilleur - seul moyen de faire coïncider son utilité de citoyen et sa gloire. Enthousiaste, enflammé par les propos du maître, il voulait rivaliser avec lui ; on reconnaît là le fruit de la " mâle éloquence ». Il n'est pas à exclure que par ce giovinetto, Solomos rende un hommage indirect à son ami Ludovico Strani, qui fut aussi un ami d'enfance de Foscolo.

Vers la fin de l'Oraison, évoquant les derniers instants de la vie du grand homme, Solomos se plaît à composer une fiction, une "fantaisie vraisemblable» (una immaginazione verosimile) où il met en scène un personnage qui, à nouveau, est probablement L. Strani. À supposer que quelqu'un ayant eu la chance et l'honneur de connaître Foscolo depuis l'enfance ait pu traverser terres et mers pour s'approcher de son lit de mort, il l'aurait certainement entendu tenir le discours désabusé - saisissant tableau de l'époque - que voici : Ami affligé, l'affliction est le compagnon le plus fidèle des hommes sur cette terre, et la mort est la meilleure des choses. À 52 ans révolus, ayant ressenti pendant quarante ans brûler en son sein la flamme de la justice, il voit maintenant en elle ce qui lui faisait chercher la solitude aux rivages comme aux sommets des montagnes de Zante, celle qui l'a poussé en Italie et lui a permis de consacrer de longues nuits à l'étude de ceux qui ont voulu par leur génie porter secours aux mortels, et chez qui il puisa la force de quitter la solitude et d'aller à la rencontre du vaste monde. Mais le spectacle du monde était, hélas, conforme à ce que la sagesse de la Bible ne faisait que trop crier : il vit un conflit incessant d'intérêts, d'opinions et d'épées; il vit les lois s'opposer aux intérêts égoïstes et les intérêts égoïstes s'opposer aux lois; l'arbitraire des lois engendrer la tyrannie et l'arbitraire des opinions engendrer licence et corruption; et un peuple, qui se disait civilisé et déclarait vouloir un monde libre, dégainer ; la fureur des massacres transformée en danse tournoyante ; un homme s'élever très rapidement au-dessus des autres pour ensuite s'effondrer avec un fracas dont bien des siècles retentiront. Croyant lui-même que l'usage du verbe devait modérer le pouvoir de ceux qui commandent et les opinions de ceux qui obéissent, il se mit à son service, mais cela assombrit et inquiéta les puissants, et une foule de mesquins surgit en travers de sa route, réclamant des louanges sans trouver satisfaction; grande, dès lors, fut leur haine. Il vit encore la folie sous les traits de la sagesse, dans les maisons et les rues, sur les places publiques, dans les académies, les palais des riches, les cabanes des pauvres; il vit la bêtise prétentieuse triompher, la scélératesse nourrir la crainte, la fortune adorée, le vil mensonge, l'ingratitude et la trahison partout honorés, la bonté bafouée, et à ce spectacle il poussa de hauts cris. Il vit encore les lettres, les lettres sacrées, n'avoir d'autre effet sur la plupart des gens que de changer l'objet de leurs passions, et cependant des lettrés continuer à les alimenter d'inepties, d'impostures, de rancunes. Partout une confusion, une oppression, un tumulte, un état de guerre troublant fortement l'esprit de l'observateur qui y trouve ample matière à méditation. Les yeux finalement tournés vers le soleil - ce ministre suprême de la nature - et les torrents de lumière qu'il déverse indifféremment sur les villes populeuses comme sur les étendues désertes, il lui sembla voir en lui l'image de la sainte Liberté voulue par Dieu. Éprouvant de la peine à raconter ce qu'il vit alors, il peut néanmoins dire que ce spectacle et la tristesse qui l'ont saisi, les cris qu'il a poussés, ont précipité l'extinction de la flamme qui le dévorait. Trêve de lamentations : au seuil de la mort, il lui reste encore assez de force pour lever ses mains nues vers 
Dieu, le remercier de cet instant, et le prier de se montrer un juge clément lorsqu'il paraîtra devant Lui.

Trêve de fiction aussi pour Solomos, et retour à la réalité à l'intérieur de l'église catholique de Zante où l'Oraison fut prononcée: avec l'espoir que les vertus de l'homme, l'usage qu'il fit de son génie et le respect qu'il éprouvait pour la religion auront fait oublier ses défauts auprès de l'éternelle Bonté et l'auront poussé dans les bras " Di Lui, che eterna ciò che a Lui somiglia " ${ }^{6}$, une apostrophe à l'esprit bienheureux de Ugo Foscolo vient clore l'Éloge. Son jeune compatriote le prie de baisser les yeux pour voir les citoyens de sa ville natale, tous réunis autour du catafalque vide, accourus en foule pour le pleurer dans la douleur de l'avoir perdu. Qu'il jette un regard sur la branche de laurier qu'une main (celle de Solomos?) a coupée au mur solitaire d'une chapelle dans la campagne de Zante, au coucher du soleil - moment propice pour penser à lui - maintenant posée sur le cercueil en hommage à tout ce dont ses compatriotes se sentent redevables envers lui. Et s'il veut combler les souhaits de cette foule de jeunes, vieux, pauvres, riches, nobles et «popolani », lettrés et illettrés, particuliers et magistrats, laïcs et prêtres qui, rassemblés dans l'église, multiplient les signes de la tête et des mains pour demander à l'orateur de proférer la prière que voici, qu'il daigne le seconder (Orazione, 1979, 207) :

Oh! Esprit immortel, au nom de cet amour que tu portas à toutes les choses excellentes, approche-toi du trône du Tout-puissant, prosterne-toi, les lèvres contre l'escabeau de ses pieds, et si aucune loi du Paradis n'interdit les larmes, prie-Le en pleurs et par des cris d'envoyer à la Patrie voisine la Liberté.

Aussi émouvante que soit cette scène finale, on est en droit de s'interroger sur la vraisemblance de cette foule comme image de la totalité harmonieuse de la population de l'île, et sur sa capacité de compréhension du texte prononcé par Solomos. Seuls quelques-uns de ses semblables, son réseau d'amis, une petite élite locale de nobles et de bourgeois ayant étudié dans les universités italiennes, devaient être en mesure de suivre la brillante rhétorique qui se déployait dans ce discours truffé d'allusions littéraires et d'un style très élevé. Comparée à l'autre scène de foule, celle de la leçon inaugurale de Foscolo à Pavie - bien réelle, celle-là - la belle fiction de Solomos relève du fantasme et donne toute la mesure de l'inadéquation de son projet à la réalité ambiante du moment.

\section{BIBLIOGRAPHIE}

BRIGHENTI Carlo, 1929, Dionisio Solomòs, Elogio di Ugo Foscolo, Ferrara : Estense.

DUBY Georges, 1974, « Histoire sociale et idéologie des sociétés », in J. LE GOFF \& P. NORA (dir.), Faire de l'histoire, Paris : Gallimard, p. 201-228.

FAURIEL Claude, 1824, Chants populaires de la Grèce moderne, tome I, Paris : Chez Firmin Didot père et fils. 
FAURIEL Claude, 1825, Chants populaires de la Grèce moderne, tome II, Paris : Chez Firmin Didot père et fils \& Dondé-Dupré père et fils.

KAPSASKIS Sokratis, 1998, Éléments de biographie de Dionysios Solomos (en grec). G. Dardanos :

Athènes.

PERETTI Joseph, 1957, Solomos. Éloge de Foscolo et autres textes, traduits de l'italien, Athènes : Collection de l'Institut français d'Athènes.

POLITIS Linos, 1991, Euvres complètes de Dionysios Solomos (en grec), tome 3, « Correspondance », Athènes : Icaros.

POLITIS Linos, 1960, Euvres complètes de Dionysios Solomos (en grec), tome 2, « Annexe ", Textes en italien (poèmes et proses). Athènes : Icaros.

Politis Linos, 1955, CEuvres complètes de Dionysios Solomos (en grec), tome 2, « Proses et œuvres en italien ", Athènes : Icaros.

POLYLAS Jacques, 1859, Euvres subsistantes de Dionysios Solomos (en grec), Corfou : Ermis, A. Terzakis.

RIzo NÉRoulos Jacovacki, 1827, Cours de littérature grecque moderne, Genève : Abraham Cherbuliez.

\section{NOTES}

1. C'est nous qui traduisons de l'original italien ou grec.

2. Niccolò Ugo Foscolo, né à Zante le 6 février 1778, aîné des quatre enfants d'Andrea Foscolo et de la Zantiote Diamantina Spathis, mourut dans le village de Turnham Green, près de Londres, et fut enterré au cimetière voisin de Chiswich. Ses ossements furent transportés à Florence en 1871, à la place qu'ils méritaient dans les « sepolcri » de Santa Croce. À Zante, Foscolo avait passé son enfance et une partie de son adolescence. Il la quitta définitivement vers la fin de l'année 1792, à l'âge de quatorze ans ; dès lors, elle devint un lieu mythique dans sa poésie et sa mémoire.

3. La rixe qui opposa les paysans de l'île - accourus pour soutenir la flotte grecque attaquant un bateau de la flotte turque qui avait accosté dans le golfe de Laganas - aux forces armées anglaises voulant s'interposer, se solda par plusieurs victimes des deux côtés et constitue un épisode dramatique qui marqua pour longtemps les consciences, à cause de la dureté des représailles des autorités.

4. «Parmi les sources documentaires les plus accessibles, et celles dont l'enseignement est le plus clair, figurent évidemment tous les écrits de propagande, les traités de bonne conduite, les discours édifiants, les manifestes, les pamphlets, les sermons, les éloges, les épitaphes, les biographies de héros exemplaires, en somme toutes les expressions verbales qu'un milieu social donne des vertus qu'il révère et des vices qu'il réprouve, et qui lui servent à défendre et à propager l'éthique où prend appui sa bonne conscience ».

5. La métaphore de l'«aiuola-terre ", qui remonte à Boèce, a une forte connotation dantesque (la Divina commedia, Paradiso XxII, 145-153), et marque l'attachement au sol natal.

6. Il s'agit d'un vers du poème d'Alessandro Manzoni In morte de Carlo Imbonati, cité ici de mémoire par Solomos. 


\section{RÉSUMÉS}

Ugo Foscolo, né en 1778 à Zante, mourut en 1827 en Angleterre, où il s'était exilé dès 1816. Ses compatriotes, lors d'une cérémonie dans la cathédrale catholique de la ville, ont rendu hommage au grand homme qui, par ses écrits et son engagement de citoyen, s'était distingué dans les lettres italiennes aussi bien que dans le domaine public d'un continent européen marqué par les bouleversements de la Révolution française, des guerres napoléoniennes et de la Restauration. Son oraison funèbre, un Éloge composé selon les règles de la rhétorique classique - et en italien fut prononcée par Dionysios Solomos devant un catafalque vide. Le choix de l'orateur, déjà connu pour son Hymne à la Liberté, et le portrait de l'homme excellent qu'il brossa dans son Éloge, offrent l'occasion d'observer les ambitions d'excellence de Solomos lui-même, ainsi que les attentes de son milieu dans l'Heptanèse alors sous mandat britannique.

Ugo Foscolo was born in Zakynthos in 1778 and died in 1827 in England, where he had been in self-exile since 1816 . His compatriots paid tribute to him with a ceremony in the Catholic Church of the city. They paid homage to the great man who distinguished himself in Italian Letters as well as with his commitment and involvement in the public sector of a European Continent rocked by the upheavals of the French Revolution, the Napoleonic wars and the Restoration. The funeral oration, a Eulogy, composed in Italian, according to the rules of classical rhetoric, was delivered by Dionysios Solomos in front of an empty coffin. The choice of the orator, already known by his Anthem (Hymn) to Liberty, and the portrait of the man he painted in his eulogy, provide an occasion to observe Solomos's own ambitions to excellence as well as the expectations of his milieu in the Heptanese, which was under British rule at the time.

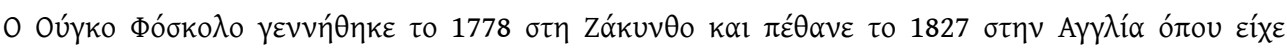

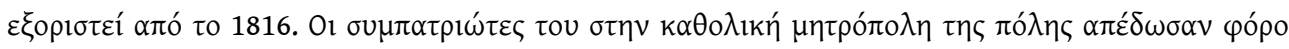

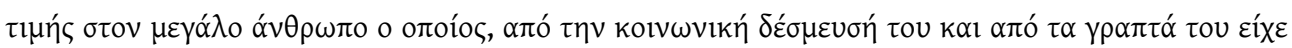

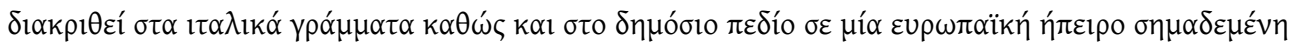

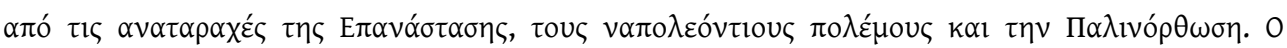

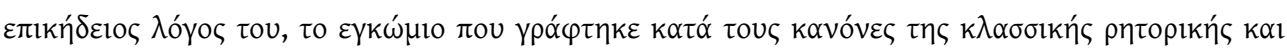

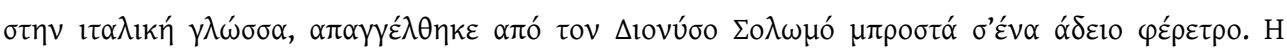

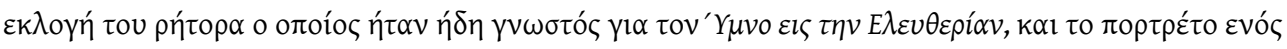

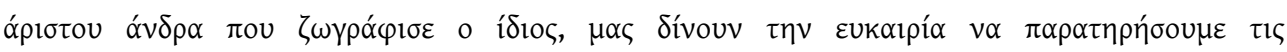

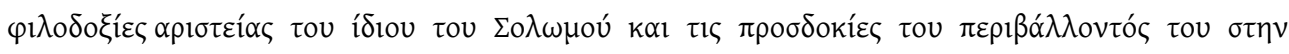

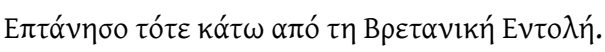


INDEX

Index géographique : Grèce

motsclestr Solomos Dionysos (1798-1857), Foscolo Ugo (1778-1827)Yunanistan, Ondokuzuncu Yüzyıl, Edebiyat, şiir

Keywords : Solomos Dionysos (1798-1857), Foscolo Ugo (1778-1827), Greece, Nineteenth century, Literature, Poetry

Mots-clés : Solomos Dionysos (1798-1857), Solomos Dionysos (1798-1857), Foscolo Ugo

(1778-1827), Foscolo Ugo (1778-1827)

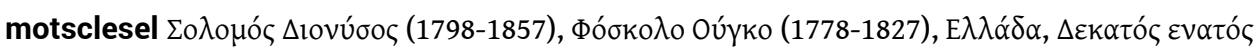

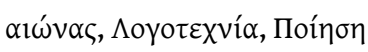

motsclesmk СОЛОМОС ДИОНИСИЈ (1798-1857), ФОСКОЛО УГО (1778-1827) ГРцИЈА,

ДЕВЕТНАЕСЕТТИОТ ВЕК, ЛИТЕРАТУРА, ПОЕЗИЈА

Thèmes : Littérature, Poésie

Index chronologique : dix-neuvième siècle

\section{AUTEUR}

\section{ANASTASIA DANAÉ LAZARIDIS}

Université de Genève 